\title{
Langmuir
}

This document is confidential and is proprietary to the American Chemical Society and its authors. Do not copy or disclose without written permission. If you have received this item in error, notify the sender and delete all copies.

\section{Molecular Simulation of the Adsorption of Methane in Engelhard Titanosilicate Frameworks}

\begin{tabular}{|r|l|}
\hline Journal: & Langmuir \\
\hline Manuscript ID: & la-2014-01554v.R1 \\
\hline Manuscript Type: & Article \\
\hline Date Submitted by the Author: & $\mathrm{n} / \mathrm{a}$ \\
\hline Complete List of Authors: & $\begin{array}{l}\text { Pillai, Renjith; Universidade de Aveiro, Departamento de Química } \\
\text { Gomes, José; CICECO - Laboratório Associado, Departamento de Química } \\
\text { Jorge, Miguel; University of Strathclyde, Department of Chemical and } \\
\text { Process Engineering }\end{array}$ \\
\hline
\end{tabular}

\section{SCHOLARONE ${ }^{\text {m }}$ \\ Manuscripts}




\title{
Molecular Simulation of the Adsorption of Methane in Engelhard
}

\section{Titanosilicate Frameworks}

\author{
Renjith S. Pillai ${ }^{1}$, José R. B Gomes ${ }^{1}$ and Miguel Jorge ${ }^{2, *}$ \\ ${ }^{1}$ CICECO, Departamento de Química, Universidade de Aveiro, Campus Universitário de Santiago, 3810-193 \\ Aveiro, Portugal \\ ${ }^{2}$ Department of Chemical and Process Engineering, University of Strathclyde, 75 Montrose Street, Glasgow G1 1XJ, \\ United Kingdom; Email: miguel.jorge@ @strath.ac.uk
}

\begin{abstract}
:
Molecular simulations were carried out to elucidate the influence of structural heterogeneity and of the presence of extra-framework cations and water molecules on the adsorption of methane in Engelhard titanosilicates, ETS-10 and ETS-4. The simulations employed three different modeling approaches, i) with fixed cations and water at their single crystal positions, ii) with fixed cations and water at their optimized positions, and iii) with mobile extra-framework cations and water molecules. Simulations employing the final two approaches provided a more realistic description of adsorption in these materials, and showed that at least some cations and water molecules are displaced from the crystallographic positions obtained from single crystal data. Upon methane adsorption in the case of ETS-10, the cations move to the large rings, while in the case of ETS-4, the water molecules and cations migrate to more available space in the larger 12-membered ring channels for better accommodation of the methane molecules. For ETS-4, we also considered adsorption in all possible pure polymorph structures and then combined these to provide an estimate of adsorption in a real ETS-4 sample. By comparing simulated adsorption isotherms to experimental data, we were able to show that both the mobility of extra-framework species and the structural heterogeneity should be taken into account for realistic predictions of adsorption in titanosilicate materials.
\end{abstract}

Keywords: Grand Canonical Monte Carlo; Gas Adsorption; Titanosilicate; Adsorption isotherm 


\section{Introduction}

The selection of an adsorbent with requisite adsorption capacity and selectivity is key to the overall efficiency and economics of an adsorption process ${ }^{1,2}$. In the last two decades, various efforts have been made for the development of efficient landfill and natural gas purification materials, and thus several authors have studied the adsorption properties of different adsorbents for natural gas upgrading ${ }^{3,4,5,6}$. Successful development of an efficient adsorbent for methane separation would allow the utilization of natural gas sources that remain unutilized due to high nitrogen content and lack of an economically viable separation methodology ${ }^{4}$. Titanosilicate materials have been emerging as attractive candidates for adsorptive gas separation and heterogeneous catalysis processes. $\mathrm{Kuznicki}^{7}$ reported in 1989 the synthesis of new microporous zeolite-type titanium silicates, namely Engelhard titanosilicates ETS-4 and ETS-10, and they have since become the focus of researchers' attention due to their interesting molecular sieving properties. The pore openings of titanosilicate ETS-4 can be thermally controlled, with the crystal lattice contracting at elevated temperatures in what is called the "molecular gate effect" 8 , $9,10,11,12$. This can improve its adsorption properties and enhance its potential for natural gas purification. In fact, these materials were already tested experimentally for the separation of methane from landfill gas by changing the extra-framework cations and also controlling the pore opening by heat treatment ${ }^{13,14,15,16,17}$. ETS-10 and ETS-4 have also been applied as potential heterogeneous base catalysts ${ }^{18,19}$, and in the stabilization of radical cations produced by photoirradiation of organic molecules adsorbed in the pores ${ }^{20}$. Very recently, the properties of ETS-10 and ETS-4 for storage and release of gaseous NO have been studied by several experimental approaches and by density functional theory (DFT) $)^{21}$. 
Titanosilicates have interesting crystalline structures formed by orthogonal chains of corner-sharing $\mathrm{TiO}_{6}$-octahedra linked by $\mathrm{SiO}_{4}$-tetrahedra through bridging oxygen atoms, generating a regular three dimensional arrangement of 12-, 8- and 7-membered ring (MR) channels. ETS-10 is a large pore molecular sieve comprising a pore opening of $8 \AA$, whereas the pores in ETS-4 have sizes of only 4-6 $\AA$. The presence of -Ti-O-Ti- wires gives optical properties to ETS materials, while the presence of channels provides them with molecular sieving capacity. The presence of tetravalent titanium in octahedral coordination generates a formal negative charge of $-2 e$, which is balanced by exchangeable cations like sodium or potassium.

Although there have been numerous experimental studies of adsorption of gases like $\mathrm{H}_{2}$, $\mathrm{N}_{2}, \mathrm{CO}_{2}, \mathrm{O}_{2}, \mathrm{Ar}, \mathrm{Xe}, \mathrm{NO}, \mathrm{CH}_{4}, \mathrm{C}_{2} \mathrm{H}_{6}$, and $\mathrm{C}_{2} \mathrm{H}_{4}$ in ETS materials ${ }^{7,9,15,16,17,22,23,24,25,26}$, the detailed mechanism of adsorption in these frameworks is not yet fully understood, particularly with regards to the interactions between adsorbates and mobile extra-framework species (not only cations, but also strongly adsorbed water molecules that may be present to stabilize the framework). Molecular simulations are important tools for revealing the microscopic interactions that have a significant impact on macroscopic properties of adsorbents like the adsorption capacity, selectivity, diffusion and molecular sieving. These techniques have previously been successful in addressing several unanswered questions in the case of zeolites, namely, distribution of cations and their availability to gas molecules, preferable adsorption sites, and diffusive properties of adsorbates ${ }^{27}$. This constitutes valuable information for the development of new materials for adsorption applications. However, there have been very few previous studies that attempted to model adsorption isotherms of methane in ETS materials. To our knowledge, the earliest studies were carried out by Nenoff and co-workers using the grand canonical Monte 
Carlo (GCMC) simulation technique to examine the suitability of ETS-10, ETS-4 and zorite for separating binary mixtures of hydrogen/methane and hydrogen/carbon dioxide ${ }^{28,29}$. Their simulations did not include any extra-framework cations or any electrostatic interactions between different species. More importantly, they did not compare their predicted adsorption isotherms ${ }^{28}$ and selectivities ${ }^{29}$ to experimental data. Siperstein et al. ${ }^{25}$ carried out GCMC simulations of adsorption of nitrogen and methane on ETS-4. Once again, they did not include extra-framework cations or electrostatic interactions in their model, and although they considered three different polymorph structures, they did not cover the entire range of structure variability. Nevertheless, their predicted adsorption isotherms for the two gases were in reasonable agreement with experimental data ${ }^{14,23}$. Recently, Nalaparaju et al. ${ }^{30}$ have presented a very detailed study of water and methane adsorption in ETS-10, taking into account electrostatic interactions and mobile extra-framework cations. By combining GCMC with molecular dynamics (MD) they were able to analyze cation mobility in this material. However, they did not compare their adsorption predictions to experimental data.

In this work, we carry out detailed GCMC simulations of adsorption of methane in both ETS-10 and ETS-4, taking into account electrostatic interactions, extra-framework species (Na cations in both materials and water in ETS-4) and a variety of possible polymorph structures to sample the degree of variability existing in real experimental samples. We directly compare simulated isotherms with experimental data and clarify the effects of cation and/or water mobility upon methane adsorption. We also show that good agreement between simulation and experiment can be obtained for ETS-4 if structural variability is taken into account. This study represents a step forward in our ability to model adsorption phenomena in titanosilicates, paving the way for computational design of these materials. 


\section{Computational Methods}

The coordinates of the framework atoms and unit cell parameters for titanosilicate materials were obtained from experimental crystallographic data by Wang and Jacobson ${ }^{31}$ for ETS-10 and by Nair et al. ${ }^{11}$ for ETS-4, and the corresponding structures are shown in Figures 14. We have used a $4 \times 4 \times 1$ arrangement of unit cells for ETS-10 (Figure 1) to generate a nearly cubic simulation cell (dimensions $29.7 \times 29.9 \times 27.08 \AA$ ) and a $2 \times 4 \times 4$ arrangement for ETS-4 (dimensions $46.45 \times 28.7 \times 27.89 \AA$ ) so as to account for the degree of structural variability in this material. Periodic boundary conditions were used in all directions. Further details about the construction of the ETS-10 and ETS-4 simulation cells are provided in Supporting Information (Table S1 and Figures S1-S12).

(a)

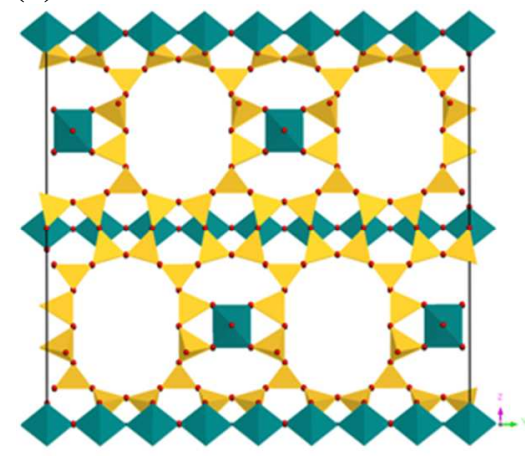

(b)

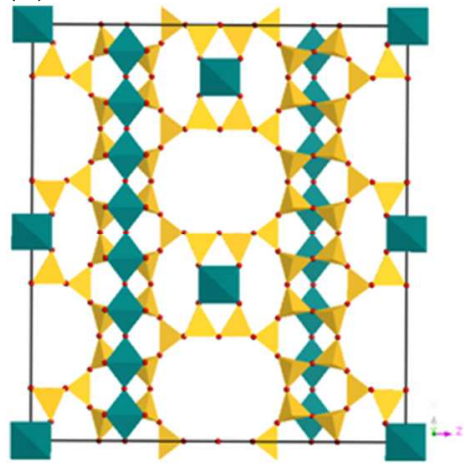

(c)

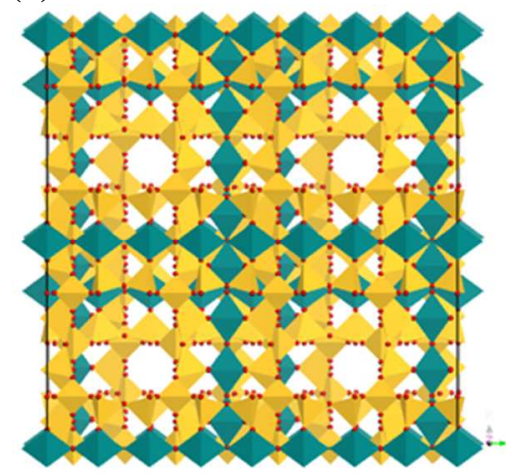

Figure 1. Projection along (a) $a$, (b) $b$ and (c) $c$ directions of the ETS-10 polymorph A simulation cell, $(4 \times 4 \times 1)$, without extra-framework cations. Color code is: bluish-green polyhedra for $\mathrm{TiO}_{6}$, yellow polyhedra for $\mathrm{SiO}_{4}$ and red for oxygen.

The ETS-4 structure can have different stackings in the $a$ and $c$ vector directions and four pure polymorphs were reported ${ }^{8}$. Essentially, the bridging units can be stacked in either ABABtype or ABCD-type sequence in the $a$ vector direction (Figure 2), whereas in the $c$ vector direction they can be stacked in either an AA- or an AC-type arrangement (Figure 3). Apart from the stacking disorder, different orientations of the apical $\mathrm{O}$ atom can be present in each 
polymorph. In fact, the crystal structure reported in the literature originates from a superposition model that considers fractional occupancies for these atoms ${ }^{11}$. We have studied two limiting cases for each polymorph: one in which 8-MR are alternately empty or occupied by two apical O atoms (Figure 4a), and another in which each 8-member ring (MR) has one apical $\mathrm{O}$ atom (Figure 4b).

(a)

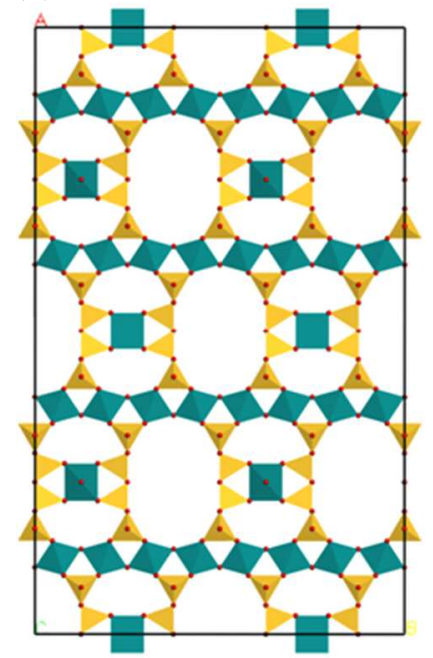

(b)

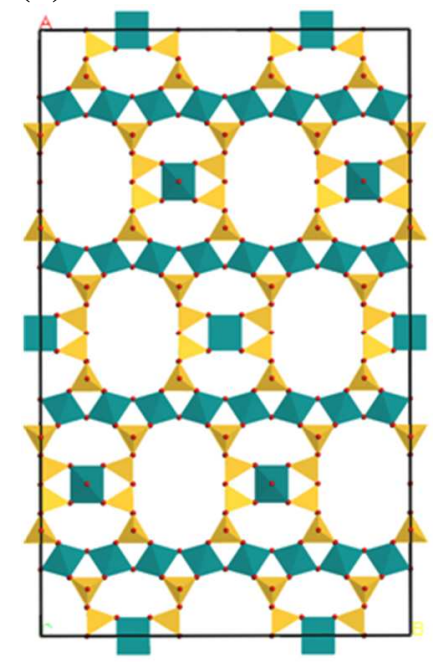

Figure 2. Periodic crystal structure of ETS-4 polymorph (a) ABAB-YY-ZZ, and (b) ABCD-YY$\mathrm{ZZ}$, viewed along $c$ vector direction. Color code as in Figure 1.

(a)

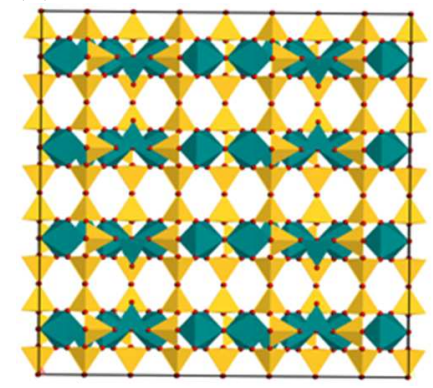

(b)

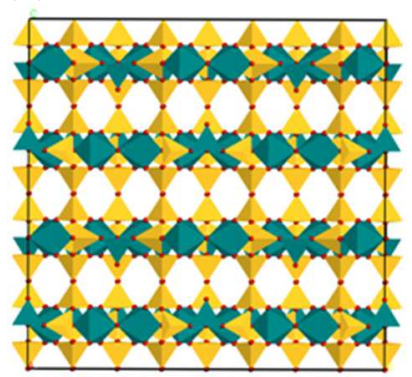

Figure 3. Periodic crystal structure of ETS-4 polymorphs, (a) XXXX-AA-ZZ and (b) XXXXAC-ZZ, viewed along $a$ vector direction. Color code as in Figure 1. 
(a)

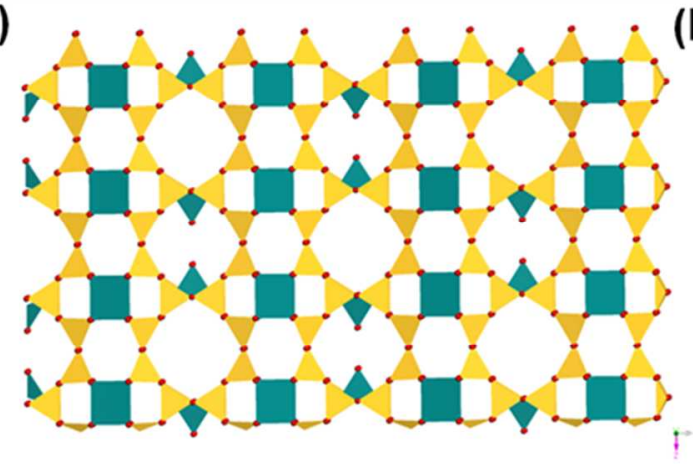

(b)

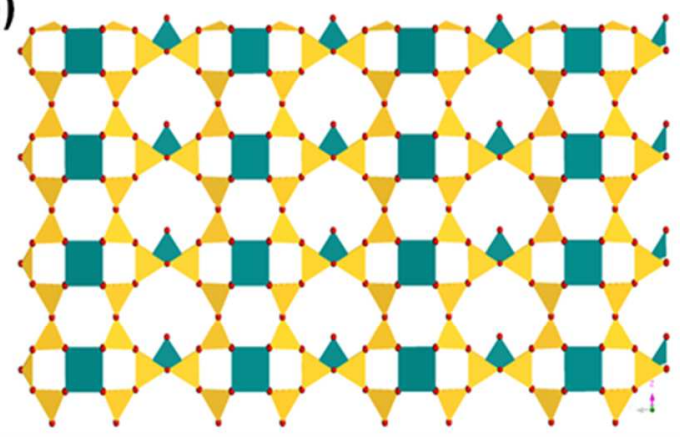

Figure 4. Periodic crystal structure of ETS-4 polymorph with alternatively blocked and vacant apical TiO in 8MR, XXXX-YY-BV (a), and half blocked apical TiO in 8MR, XXXX-YY-HB (b), viewed along $b$ direction in polymorph ABAB. Color code as in Figure 1.

In our simulations, we have taken into consideration all possible combinations of the two stacking arrangements and of the apical $\mathrm{O}$ orientations. This means that the total number of different ETS-4 structures studied in this work is eight, with the following nomenclature: XXXX-YY-ZZ, where XXXX refers to the stacking in the $a$ direction (could be ABAB or $\mathrm{ABCD}$ ), YY refers to stacking in the $c$ direction (could be AA or AC), and $\mathrm{ZZ}$ refers to the orientation of the apical oxygen atoms (could be HB for half-blocked pores or BV for alternately fully blocked and vacant pores). So, for example, $A B C D-A C-H B$ means a structure with $A B C D$ stacking along the $a$ vector (Figure 2b), AC stacking along the $c$ vector (Figure $3 b$ ) and halfblocked pores (Figure 4b). It is important to notice that the real material is likely to be composed of a combination of each of the four polymorphs, organized in domains of different size, with possibly random oxygen orientations. This degree of disorder is almost impossible to capture in a small simulation cell, so we restrict ourselves here to the study of pure polymorphs, which should be interpreted as limiting cases for the adsorption in a real ETS-4 material (see the Results and Discussion section). 
All simulations were carried out using the MUSIC code developed by the Snurr group ${ }^{32,}$ 33. The frameworks were considered to be rigid and framework atoms were kept fixed at their crystallographic positions for all kinds of simulations. Charge balancing sodium cations were added to each framework model to ensure charge neutrality. In the case of ETS-4, we also included structural water molecules. Indeed, according to the thermal gravimetry analysis (TGA) experiments of Nair et al. ${ }^{11}$ and Marathe et al. ${ }^{17}$, ETS-4 loses all non-structural water molecules on activation up to $373 \mathrm{~K}$, but the structural molecules are only lost between $373 \mathrm{~K}$ and $443 \mathrm{~K}$, after which the ETS-4 material collapses entirely. We are comparing our simulations to adsorption experiments preceded by activation at $373 \mathrm{~K}^{24}$, therefore our simulations should take into account the structural water molecules.

The LJ parameters and point charges used for the adsorbate-adsorbate and adsorbateframework interactions are listed in Table S1 and were taken from the work of Nalaparaju et al. $^{30}$. These authors used LJ parameters obtained from the universal force field (UFF) ${ }^{34}$ while the point charges for the framework $\mathrm{Ti}, \mathrm{Si}$, and $\mathrm{O}$ atoms were produced from fitting of the electrostatic potential from DFT calculations on a cluster model of ETS-10 ${ }^{30}$. The TraPPE united atom model ${ }^{35}$ was used for methane and the TIP3P potential was employed to model the water molecules ${ }^{36}$. The choice of water model was found to have a practically negligible effect on the adsorption of methane on ETS materials (see Figure S13). Lorentz-Berthelot mixing rules were used to calculate cross-site interactions. The LJ interactions were evaluated with a spherical cut-off length of $13 \AA$, while Ewald sums were used to account for long-range electrostatic interactions.

To assess the effect of the mobility of extra-framework species $\left(\mathrm{Na}^{+}\right.$cations for both materials and also water for ETS-4) during adsorption, we have adopted three different 
simulation strategies, described briefly below. Further methodological details are provided in Supporting Information.

\section{- $\quad$ Strategy 1 - Simulations with fixed cations and water at their crystallographic positions}

The crystallographic data of Wang and Jacobson ${ }^{31}$ and Nair et al. ${ }^{11}$ were used to obtain the positions of cations and water for ETS-10 and ETS-4, respectively. To determine the atomic coordinates for hydrogen atoms in water molecules occluded in ETS-4, we used the same procedure as Pinto et al. ${ }^{21}$, whereby the hydrogen atom coordinates of water molecules were optimized by periodic DFT. The crystallographic positions of extra-framework species are shown in Figure S1 for ETS-10 and Figure S2 for ETS-4, together with a more detailed description of their coordination environment. Crystal data identified 2 different cation sites in both ETS-10 and ETS-4 (in both cases denoted as Na1 and Na2). There are four crystallographically distinct water sites in ETS-4, denoted by Ow1, Ow2, Ow3 and Ow4 in Figure S2. These water molecules are the structure directing molecules in ETS-4, and are needed to maintain the structural integrity of the framework ${ }^{11,17}$. It is important to note that the probability to find 8-MRs without water molecules seems to be extremely low and, therefore, the 8 -MR is either fully or partially blocked by water molecules ${ }^{11,12}$. Once the structures were built, adsorption isotherms were computed with fixed cation and water positions using the GCMC algorithm for both materials at several temperatures, in each case employing 7 million Monte Carlo steps.

\section{- $\quad$ Strategy 2 - Simulations with cations and water fixed at optimized positions}

In this approach, we also computed adsorption isotherms by GCMC with fixed cations and water, but in this case we previously allowed the extra-framework species to equilibrate during a long (7 million steps) NVT simulation in the empty framework at $300 \mathrm{~K}$. During these 
NVT simulations, cations and water were allowed to move by way of MC translation trials. The most favorable positions for the extra-framework species were calculated by averaging their positions in 3000 equilibrated frames, evenly spaced throughout the final sampling period of the NVT run. We have also plotted occupancy maps for water and cations to provide a visual assessment of the most favorable positions for the extra-framework cations and of their thermal fluctuations. Further details are reported in Supporting Information. For both materials (and all different polymorphs in the case of ETS-4), we then carried out GCMC simulations of methane adsorption with cations and water molecules fixed at their most favorable positions, determined from the NVT simulations described above.

\section{- $\quad$ Strategy 3 - Simulations with mobile extra framework species}

Methane adsorption was also simulated by GCMC considering fully mobile extraframework species. For these adsorption simulations, we performed insertion, translation, rotation, and deletion moves for methane molecules, translation and rotation for water molecules, and only translation moves for cations (they are spherically symmetric, so rotation is meaningless).

\section{Results and Discussion}

\subsection{Cation distribution and mobility in ETS-10}

The occupancy maps of the sodium cations in the ETS-10 material after simulations in the NVT ensemble are compared in Figure 5 with those reported in crystallographic data ${ }^{31}$ and in previous simulation studies ${ }^{30}$. Our NVT simulations indicate five clear preferred cation positions, which are equivalent to those reported by Nalaparaju et al. ${ }^{30}$ and are thus named according to those authors' notation. The cations at sites I and II have access to guest molecules in the 12-MR channel, but they contribute weakly towards adsorption because of shielding effects by $\mathrm{O}$ atoms 
in the 5-member silica rings. Site III is located in the 12-MR channel and has less shielding from $\mathrm{O}$ atoms, thus the $\mathrm{Na}^{+}$ion at this site has a relatively strong interaction with guest molecules in the 12-MR channel. Site IV is neighboring Ti atoms in the -Ti-O-Ti-chain. Site V occupies the 12-MR channel and sits between two orthogonal-O-Ti-O-chains.

(a)

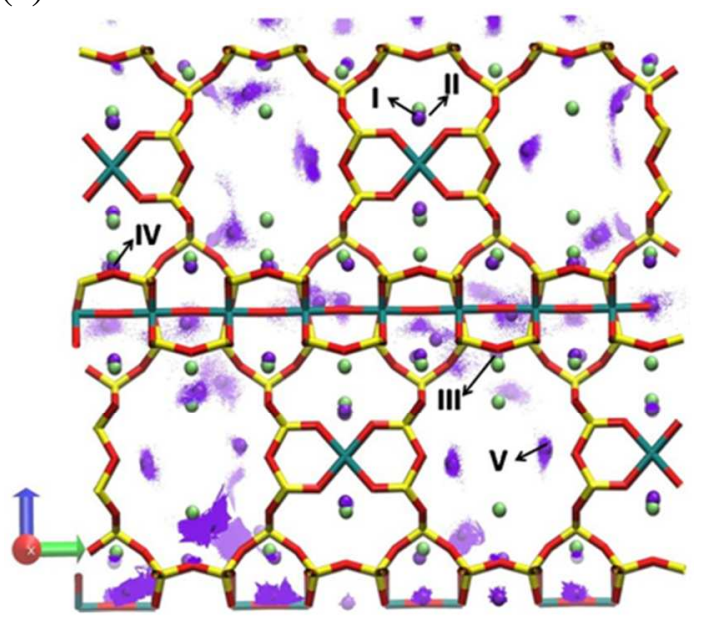

(b)

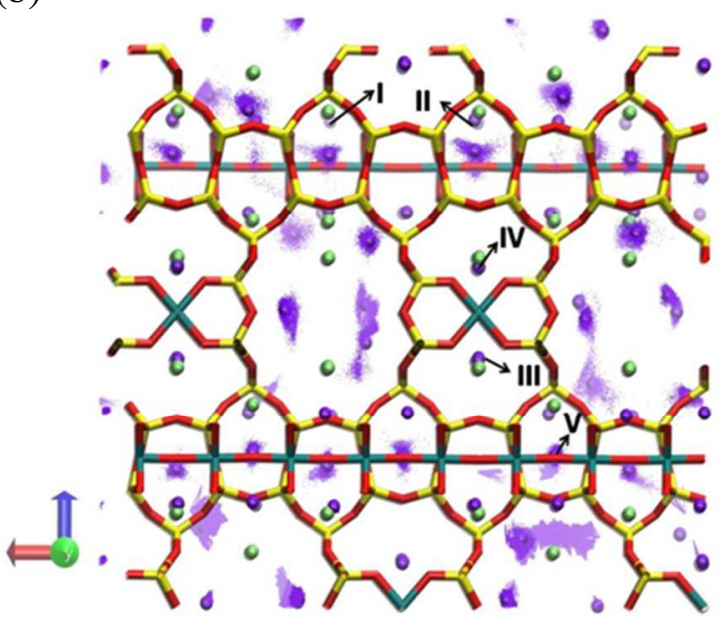

Figure 5. Comparison of sodium positions in ETS-10 obtained from experimental crystallographic positions (green spheres), instantaneous equilibrated cation locations (violet dots), and optimal cation locations (violet spheres) obtained from NVT simulation showing views along the $a$ vector direction, (a) and along the $b$ direction (b).We also show the notation for the preferred cation locations (in black), following Nalaparaju et al. ${ }^{30}$. Tubes represent the framework, with yellow for silicon, red for oxygen and bluish-green for titanium.

It is clear that there is not a perfect match between the crystallographic cation positions and the equilibrated positions. In fact, while some of the channels are occupied by cations in the single crystal structure, they are vacant in the equilibrated structure (see, e.g., the large pores in Figure 5b). Conversely, some of the most favorable cation positions from NVT simulation are not present in the crystal structure (e.g., site V). This is likely to be a consequence of the fact that crystal structure refinement often imposes excessive symmetry constraints, which poses a problem for highly mobile species such as extra-framework cations. Another interesting observation is that our NVT occupancy map shows evidence for the existence of two types of $\mathrm{Na}^{+}$motion, with some cations remaining practically fixed during the entire simulation (standard 
deviation of their location $\leq 0.5$, for types I-IV) while others are highly mobile (standard deviation as large as 3.0 for type V), and hence loosely bound to the framework. This confirms earlier observations from MD simulations of cation mobility in ETS-10 ${ }^{30}$. Both these observations are likely to have important consequences for adsorption - on the one hand, we should not expect simulations with cations fixed at their crystallographic positions to provide an accurate representation of the adsorbed phase; on the other hand, it is possible that at least some of the highly mobile cations may relocate during adsorption to better accommodate the adsorbate molecules. In the following section, we will test the importance of each of these two effects.

\subsection{Adsorption of $\mathrm{CH}_{4}$ in ETS-10}

Figure 6 compares the experimental adsorption isotherm of methane in ETS-10 at five different temperatures ${ }^{37}$ with GCMC isotherms calculated using the three different treatments of the extra-framework cations described in section 2. The simulated methane isotherms obtained from all three strategies overestimate the experimental adsorption isotherms at all temperatures and pressures. Nevertheless, it is encouraging that the curvature of the experimental isotherms is well reproduced by our simulations. The discrepancies between simulation and experiment could be due to the presence of impurities and/or structural defects in the adsorbent samples of ETS-10 used for the experiments, in contrast to the pure polymorph A unit cell used in the simulations. In fact, it is well known that real ETS-10 frameworks show a significant degree of structural disorder $^{38,39}$, while their bulk synthesis may suffer from loss of crystallinity and/or formation of other solid phases $40,41,42$. To test this hypothesis, we rescaled all the experimental isotherms by the same empirical scaling factor to maximize agreement with simulations carried out with strategy 3 (which we consider to be the most realistic, as discussed below). These rescaled 
experimental isotherms are shown as open stars in Figure 6, and demonstrate that reasonable agreement can be obtained using a scaling factor of 1.75 .

(a)

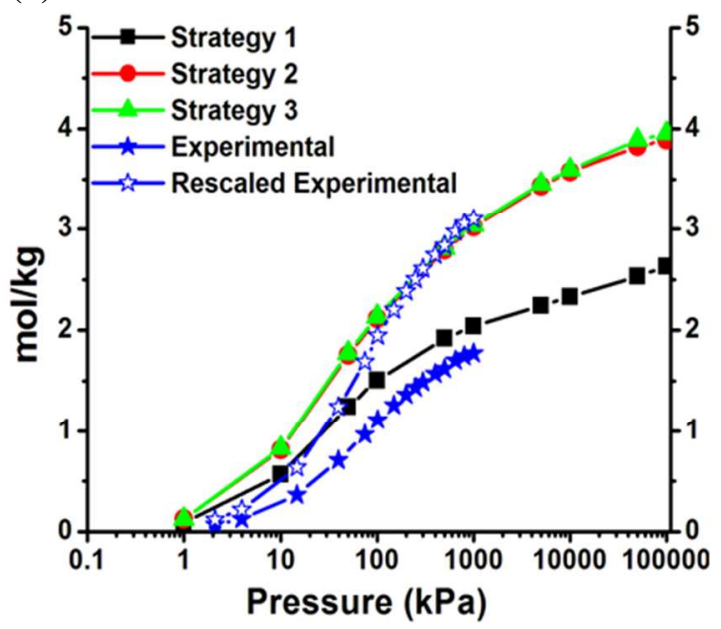

(c)

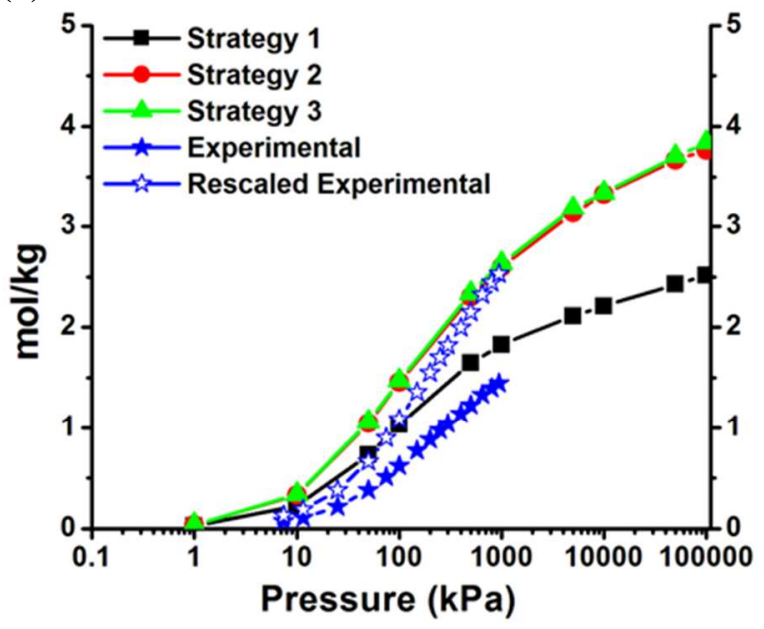

(e)

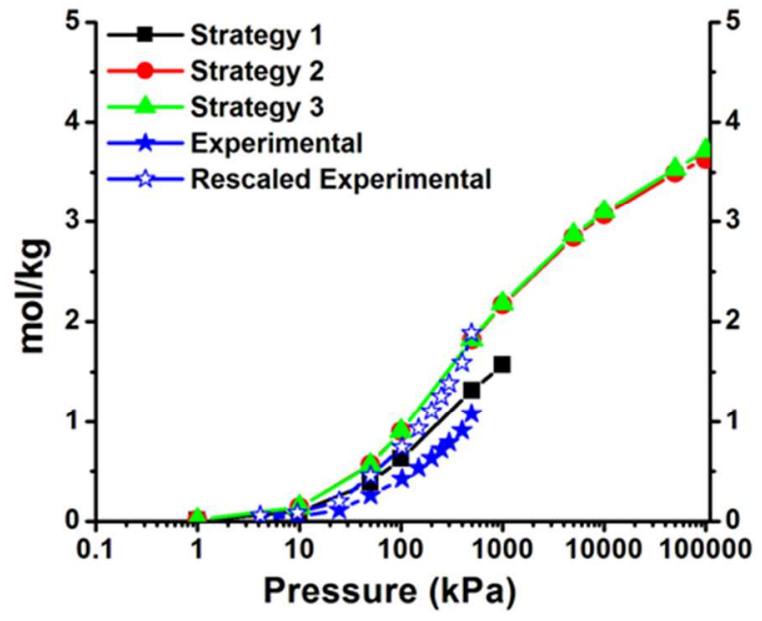

(b)

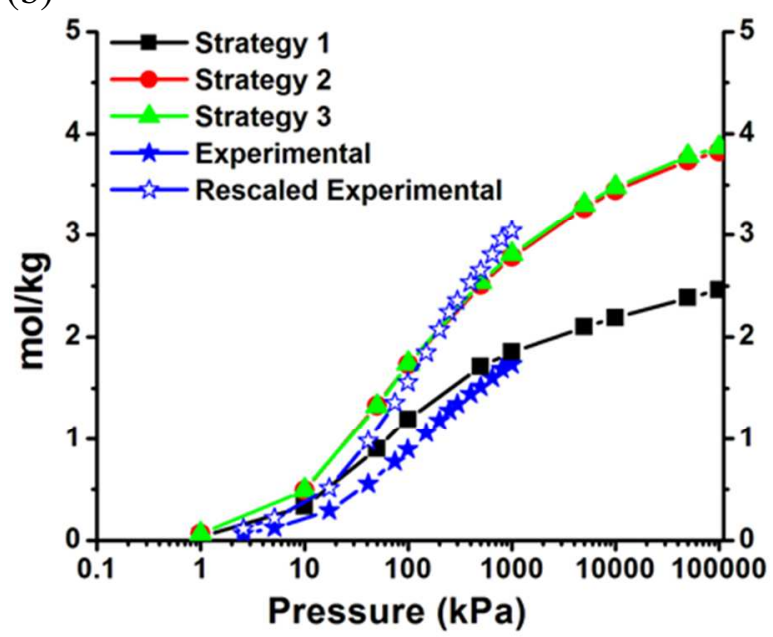

(d)

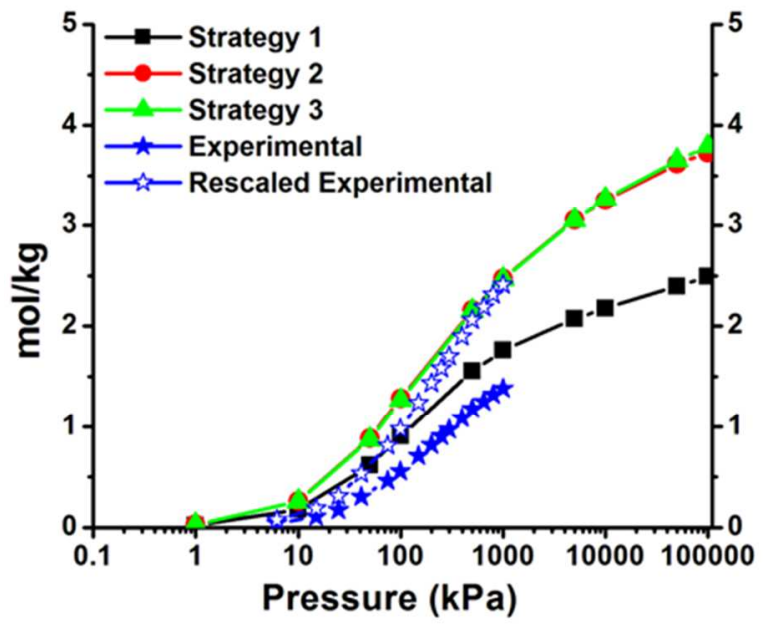


Figure 6. Experimental ${ }^{37}$ and simulated adsorption isotherms for $\mathrm{CH}_{4}$ in Na-ETS-10 at (a) 280 $\mathrm{K}$, (b) $300 \mathrm{~K}$, (c) $315 \mathrm{~K}$, (d) $325 \mathrm{~K}$ and (e) $350 \mathrm{~K}$. Simulations were performed using the three strategies described in section 2 . We also show the experimental isotherms rescaled by an empirical factor of 1.75 as blue open stars.

Recently, some of us ${ }^{43}$ proposed a more physically consistent approach to account for the effect of impurities in experimental samples of crystalline adsorbents, based on determining the scaling factor from the ratio of the theoretical pore volume of a pure crystal to the pore volume reported experimentally. The main assumption in this approach is that the only effect of impurities and defects is to reduce the available pore volume of the material. The theoretical pore volume determined from a simulated $\mathrm{N}_{2}$ adsorption isotherm at $77 \mathrm{~K}$ is $0.1472 \mathrm{~cm}^{3} / \mathrm{g}$, while the value reported by Al-Baghli et al. ${ }^{37}$ is $0.1195 \mathrm{~cm}^{3} / \mathrm{g}$. This yields a scaling factor for this sample of 1.23 , which is significantly lower than the empirical scaling factor of 1.75 . This suggests that pore volume reduction caused by impurities and/or defects in the experimental sample can only partially account for the discrepancies between simulation and experiment. Other possible sources of error are inadequacies of the molecular model, problems in the experimental adsorption measurements (e.g., insufficient equilibration time) or non-linear effects of impurities. Without further studies, we cannot presently rule out any of these hypotheses.

Figure 6 allows us to assess the effect of cation mobility on methane adsorption. It is clear that consideration of fixed cations at crystallographic positions leads to a serious underestimation of the adsorbed amount compared to the simulation with fully mobile cations. As discussed above, this is likely due to an inaccurate description of the most favorable cation positions in the crystal structure data. Figure 7 compares the occupancy maps for adsorbed methane in ETS-10 considering strategies 1 and 3. The strategy 1 simulation indicates that the majority of methane molecules are located in the centre of the 12-MR, which can be seen from 
Figure 7a. However, the simulations with both fixed cations at pre-optimized positions (strategy 2, results not shown) and with mobile cations (Figure $7 \mathrm{~b}$ ) show that there are two parallel rows of $\mathrm{CH}_{4}$ packing in the 12-MR as well as $\mathrm{CH}_{4}$ molecules close to the 7-MR along the $b$ vector view of the ETS-10 framework. In both cases, the pore space accessed by methane is much larger than with strategy 1, where the fixed cations located in the 12-MR are blocking a significant region of the pore space. This explains why strategy 1 leads to much lower adsorbed amounts at all temperatures and pressures. Interestingly, the adsorption isotherms obtained from strategies 2 and 3 are very similar (Figure 6). This suggests that for methane adsorption in ETS10, the location of the cations is more relevant than its movement. Indeed, comparison of Figures $5 \mathrm{~b}$ and $7 \mathrm{~b}$ shows that the positions of the cations in the 7-MR and 12-MR channels are practically the same upon methane sorption as in the empty framework, indicating little cation displacement caused by methane uptake. It should be noted, however, that cation displacements may play an increasingly important role in adsorption of more polar species, such as carbon dioxide and water. Further studies are needed to clarify this hypothesis.

(a)

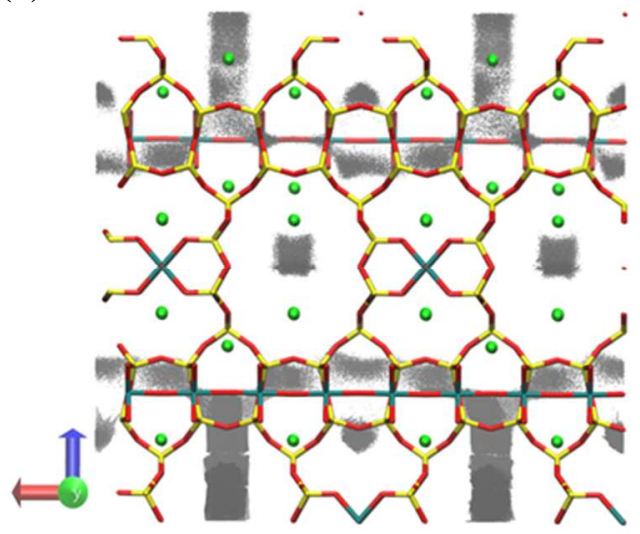

(b)

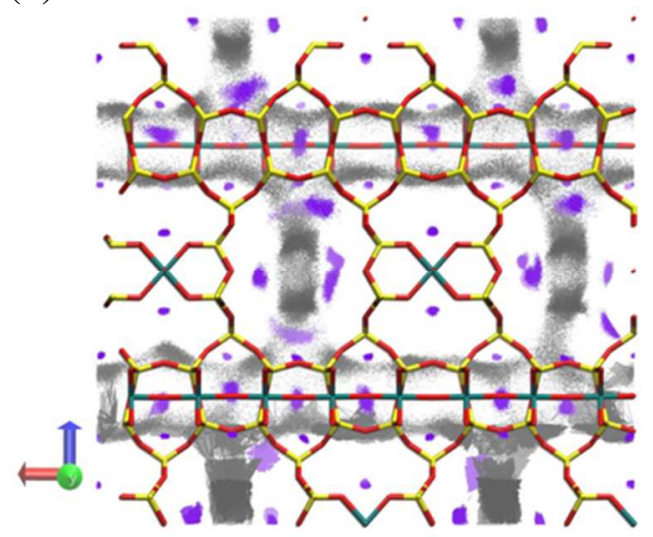

Figure 7. Occupancy maps for $\mathrm{CH}_{4}$ (gray dots) in ETS-10 viewed along the $b$ vector with (a) cations fixed at crystallographic positions (green spheres) and (b) moving cations (violet dots), at $300 \mathrm{~K}$ and $1 \times 10^{5} \mathrm{kPa}$. Color coding for the framework is the same as in Figure 5. 


\subsection{Cation and water distribution and mobility in ETS-4}

From the conclusions taken in the preceding section one expects that in the case of ETS4, not only the distribution of $\mathrm{Na}^{+}$cations inside the pores of the material but also the distribution of the water molecules will influence the adsorption of methane. Therefore, sodium cations and water molecules will have to be considered as moving species in order to obtain an accurate picture of the mechanism of methane sorption in ETS-4. Such studies are novel for ETS-4 as are the studies involving the interaction of water and extra-framework cations with the ETS-4 framework atoms.

Figure 8 shows the occupancy maps of equilibrated extra-framework water and sodium in ETS-4 obtained after NVT MC simulations at $303 \mathrm{~K}$, compared to the single crystal positions. We show only polymorph ABAB-AA-HB, for which the differences are more evident, but plots for all other idealized polymorphs of ETS-4 are reported in supporting information (Figure S6). The occupancy maps for ETS-4 again indicate that the single crystal cation positions do not provide a complete description of the most favorable equilibrium locations. Two of the most favorable positions arising from the NVT simulation are indeed similar to those in single crystal data $^{11}$, i.e. Na1 and $\mathrm{Na} 2$. However, other than these two cation positions, an extra cation location is observed parallel to the apical TiO bridged 12-MR when viewed along the $c$ vector direction (Figure 8a), and located in the 8-MR between two apical TiO when viewed along the $b$ vector direction (Figure 8c). This position is hereafter referred to as $\mathrm{Na3}$.

The favorable location maps for all polymorphs (Figure S6) indicate that there are no significant differences of cation locations between polymorphs, with the extra framework cation species being located in each polymorph according to the structural morphology obtained by different stacking of 12-MRs. However, there is a difference in occupancy for sodium in 8-MR 
due to the orientation of the apical TiO. For example, the most favorable position maps obtained in ABAB-AA-HB polymorph (cf. Figure 8) show that the extra-framework cations have good mobility in half-blocked 8-MR, each of which contains only one apical TiO. On the other hand, in structures of type XXXX-YY-BV, cations show a high degree of mobility inside vacant 8MR, which contain no apical oxygen atoms (Figures S6e-h), whereas their movement is highly constrained in fully blocked 8-MR with two apical TiO units (Figures S6a-d).

A close inspection of the occupancy maps for water in ETS-4 polymorphs (Figure S6) reveals similar water distributions in $12-$ and $8-\mathrm{MRs}$ for all polymorphs. The NVT simulations show that the most favorable locations are similar to the single crystal positions reported by Nair et $\mathrm{al}^{11}$, and no significant new locations were identified. However, it is clear that water molecules show significant thermal movement around the equilibrium positions. Thus, it is likely that water molecules, as well as some of the cations, can be displaced upon adsorption at relatively high temperatures. Therefore, the locations and mobility of the extra framework cations and water molecules may have an important influence on adsorption of guest molecules in the channels of ETS-4. In the next section, we will test this possibility by analyzing results of GCMC adsorption simulations with fixed and mobile extra-framework species. 
(a)

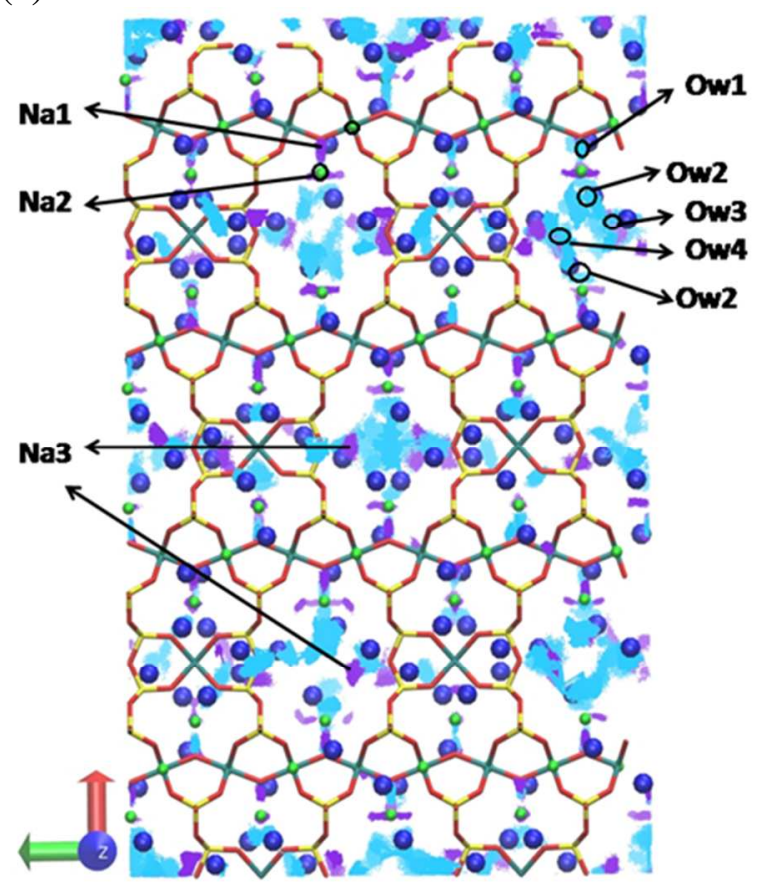

(b)

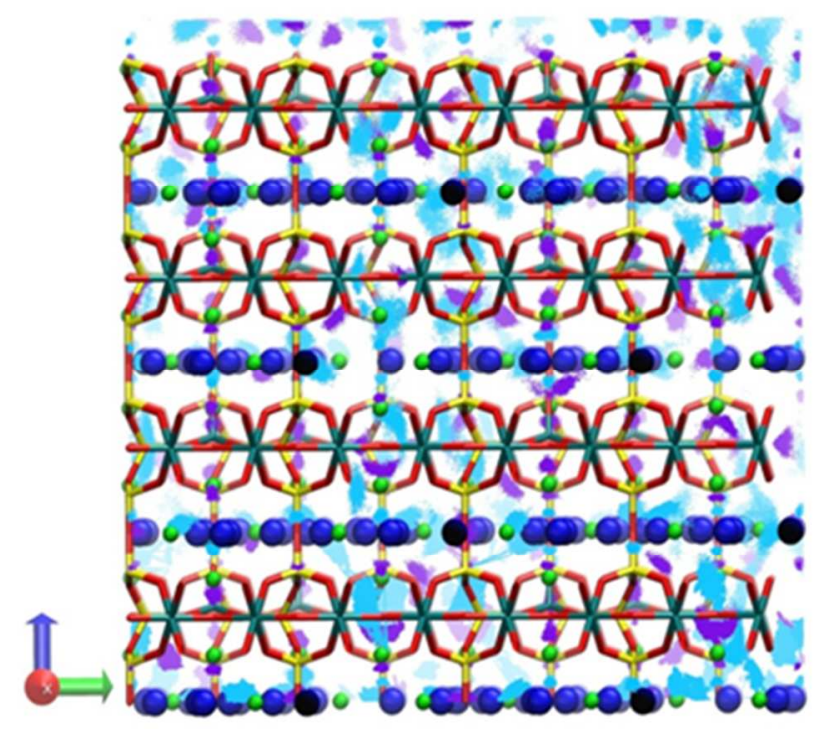

(c)

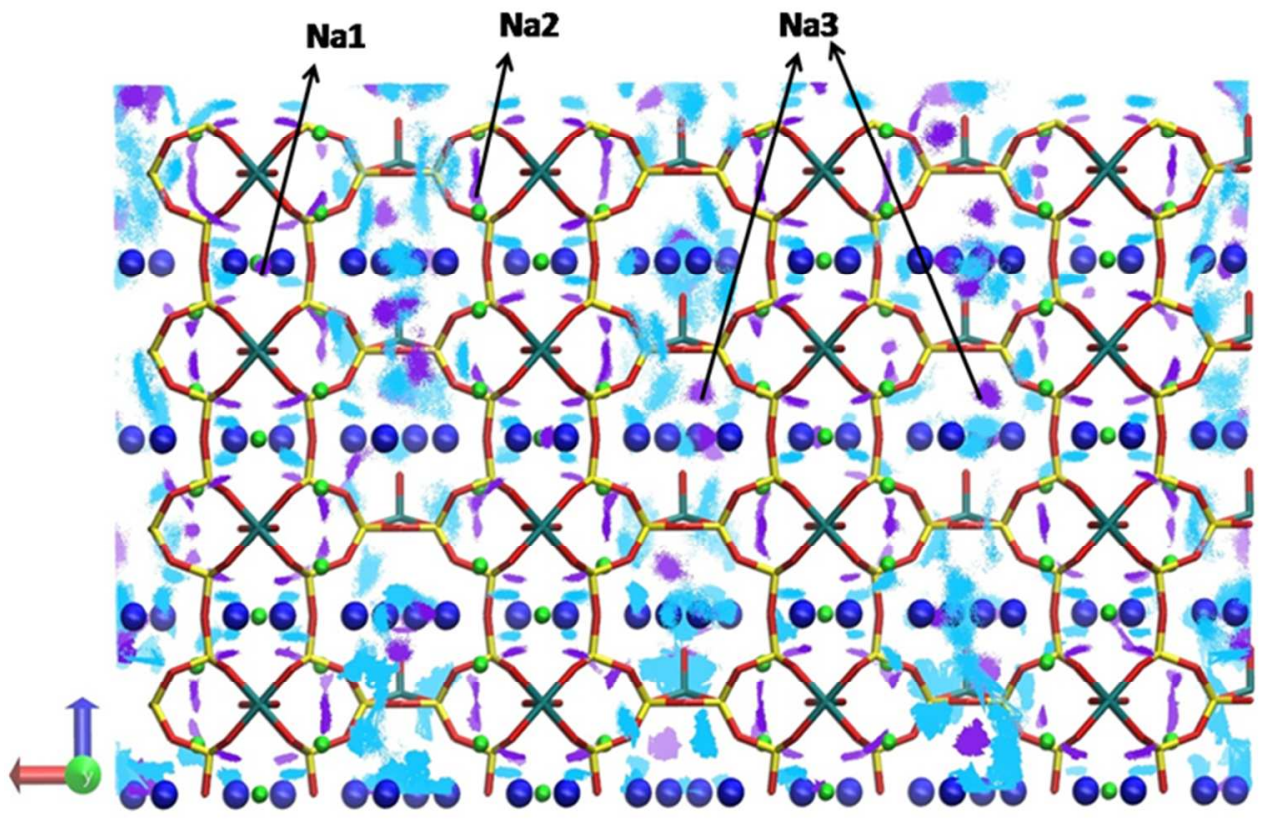

Figure 8. Comparison of sodium and water positions in ABAB-AA-HB obtained from experimental crystallographic positions (green and blue spheres for sodium and water, respectively), instantaneous equilibrated sodium cation (violet dots), and water (cyan dots) locations obtained from NVT simulation showing views along the $c(\mathrm{a}), a(\mathrm{~b})$, and $b$ (c) vectors. The new position $\mathrm{Na} 3$ is indicated by an arrow. Color coding for the framework is the same as in Figure 5. 


\subsection{Sorption of $\mathrm{CH}_{4}$ in ETS-4}

We begin by analyzing the effect of cation and water location and mobility on methane adsorption isotherms in ETS-4 through GCMC simulations using the three different strategies described in section 2. Figure 9 shows the simulated adsorption isotherms of methane at $303 \mathrm{~K}$ in the ABCD-AC-BV and ABAB-AA-HB polymorphs of ETS-4 with those three strategies. These polymorphs were chosen because they represent two typical cases regarding the effects of mobility, but the complete set of isotherms for all ETS-4 polymorphs at three different temperatures are provided in supporting information (Figures S7-S9). Several conclusions can be reached by examination of these isotherms and of the corresponding occupancy maps for methane adsorption (Figures S10-S12).

(a)

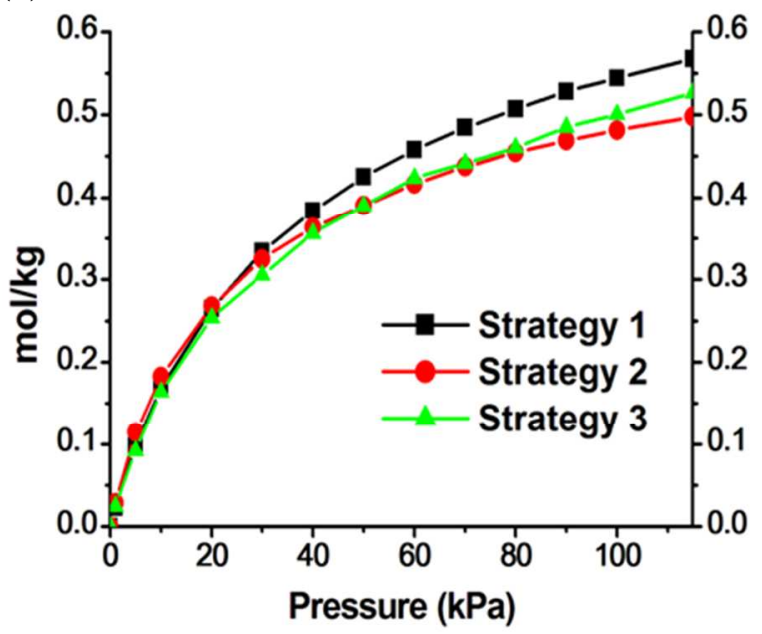

(b)

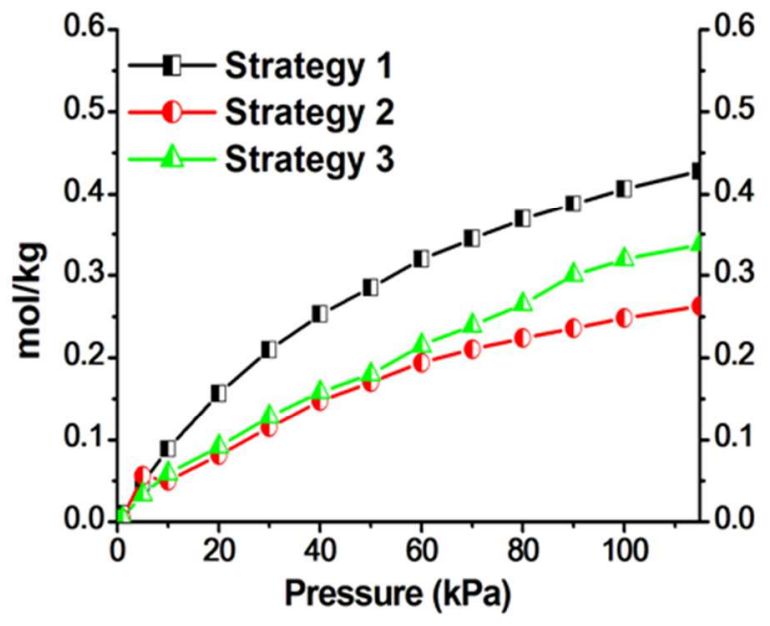

Figure 9. GCMC adsorption isotherms for $\mathrm{CH}_{4}$ at $303 \mathrm{~K}$ in ETS-4 polymorphs ABCD-AC-BV (a) and ABAB-AA-HB (b).

The first observation is that the effects of mobility practically do not depend on the stacking of the ETS-4 framework in the $a$ direction, i.e., polymorphs ABAB-YY-ZZ and ABCDYY-ZZ show essentially the same effects of cation and water mobility. This can be seen by comparing the trends in the left-hand side panels to those in the right-hand side panels in Figures 
S7-S9, which are very similar. Mobility of extra-framework species seems to have a slightly larger effect on polymorphs of type XXXX-AA-ZZ than in XXXX-AC-ZZ, although this difference is not very pronounced (compare, e.g., Figures S8a and S8b to S8c and S8d, respectively).

The most remarkable difference is between polymorphs of type XXXX-YY-BV and XXXX-YY-HB, with the latter exhibiting much more significant effects of cation and water mobility. In fact, while adsorption isotherms obtained with the three different strategies on the former structures are very similar to each other, as exemplified in Figure 9a, isotherms calculated on the latter type of structures show marked differences, evident in Figure 9b (compare also the top 4 panels to the bottom 4 panels in Figures S7-S9). This can be further interpreted by comparing the methane occupancy maps for the 3 strategies in the two types of structure, shown in Figure 10 for polymorph $\mathrm{ABAB}-\mathrm{AA}-\mathrm{BV}$ and in Figure 11 for $\mathrm{ABAB}-\mathrm{AA}-\mathrm{HB}$. In these figures, we have also highlighted the most favorable locations of extra-framework cations and water molecules. As mentioned previously, while the crystallographic data only identifies two preferred cation positions ( $\mathrm{Na} 1$ and $\mathrm{Na} 2$ ), NVT simulations reveal a third location, denoted by Na3. Apart from this, however, the water and cation positions are not very different in the three strategies for polymorph ABAB-AA-BV (Figure 10). Similarly, although it is not as easy to discern because of the large amount of scatter in the occupancy maps, the regions of the pore space occupied by adsorbed methane molecules in this polymorph do not change significantly when cations and water are allowed to move. As mentioned above, this is then reflected in very similar adsorption isotherms for polymorphs of type XXXX-YY-BV (Figure 9a). 
(a)

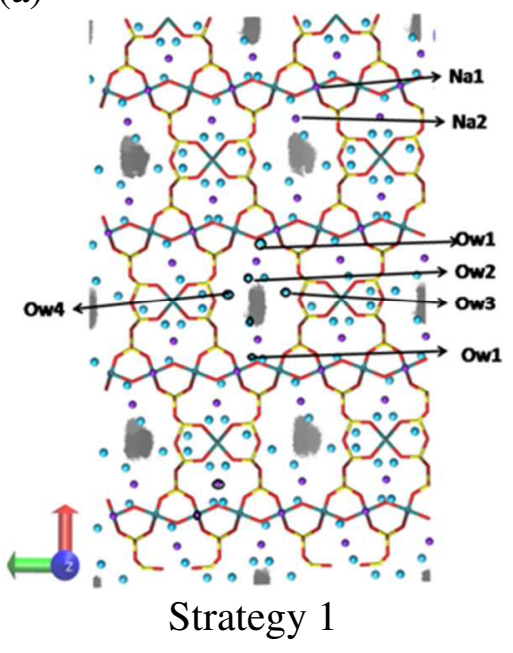

(b)

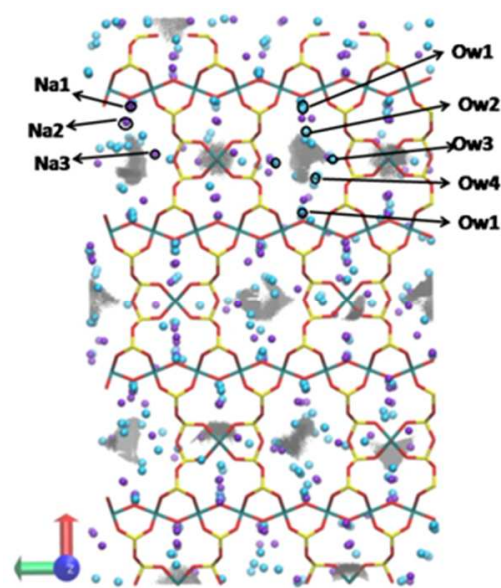

Strategy 2

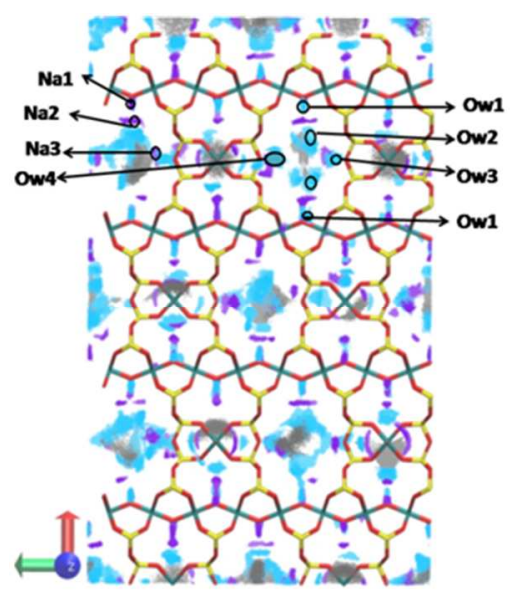

Strategy 3

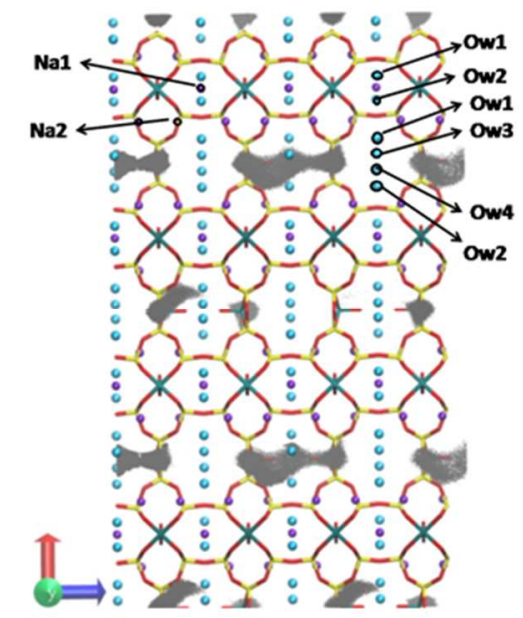

Strategy 1

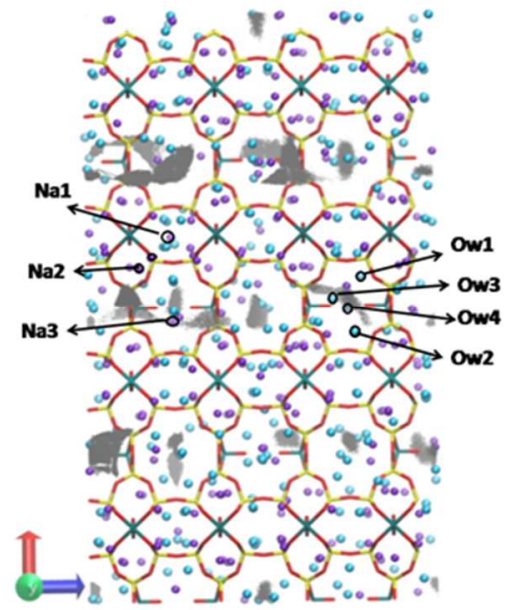

Strategy 2

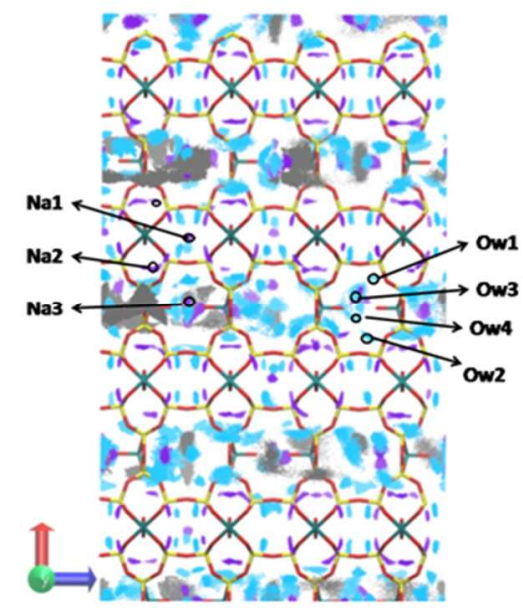

Strategy 3

Figure 10. Occupancy maps for $\mathrm{CH}_{4}$ at $300 \mathrm{~K}$ and $115 \mathrm{kPa}$ in the ABAB-AA-BV polymorph of ETS-4, shown in projections along the (a) $c$ and (b) $b$ vectors. For each direction, we compare results obtained using the three simulation strategies. Color coding is the same as in Figure 7.

In contrast, when we compare the maps obtained from the three strategies for polymorph ABAB-AA-HB (Figure 11), we observe some significant differences in the most favorable positions of cations and water (compare, e.g., the Nal positions in Figure 11) and, more importantly, in the regions of the pore space favored by methane. This is then translated in much more pronounced differences in adsorption isotherms obtained from the 3 strategies in polymorphs of type XXXX-YY-HB (Figure 9b). The different behavior of these two types of 
polymorphs can be explained by considering that in the first case the 8-MR pores are alternately vacant and fully blocked by apical oxygen atoms. Although water and cations can be present in the vacant pores, they still allow for methane molecules to adsorb in these cavities, and this is observed for all strategies. However, when all of the 8-MR pores are partially blocked by apical oxygen atoms, the presence of a few extra-framework species is sufficient to completely exclude methane from this region of the pore space. Since this does not happen for strategy 1, adsorption is highest in this case than for the two other strategies, where extra-framework species are present in the 8-MR pores. Strategy 2 generally yields lower adsorbed amounts than strategy 3 because in the latter the cations and water can dynamically adjust to the presence of adsorbed methane. As a consequence, we observe some cases in which the isotherms for strategy 3 approach those of strategy 1 at high pressure (see, e.g., Figure 9b and Figures S8f-h). In other words, we can say that in strategy 3 , methane molecules are competing with water for the most favorable adsorption sites.

(a)
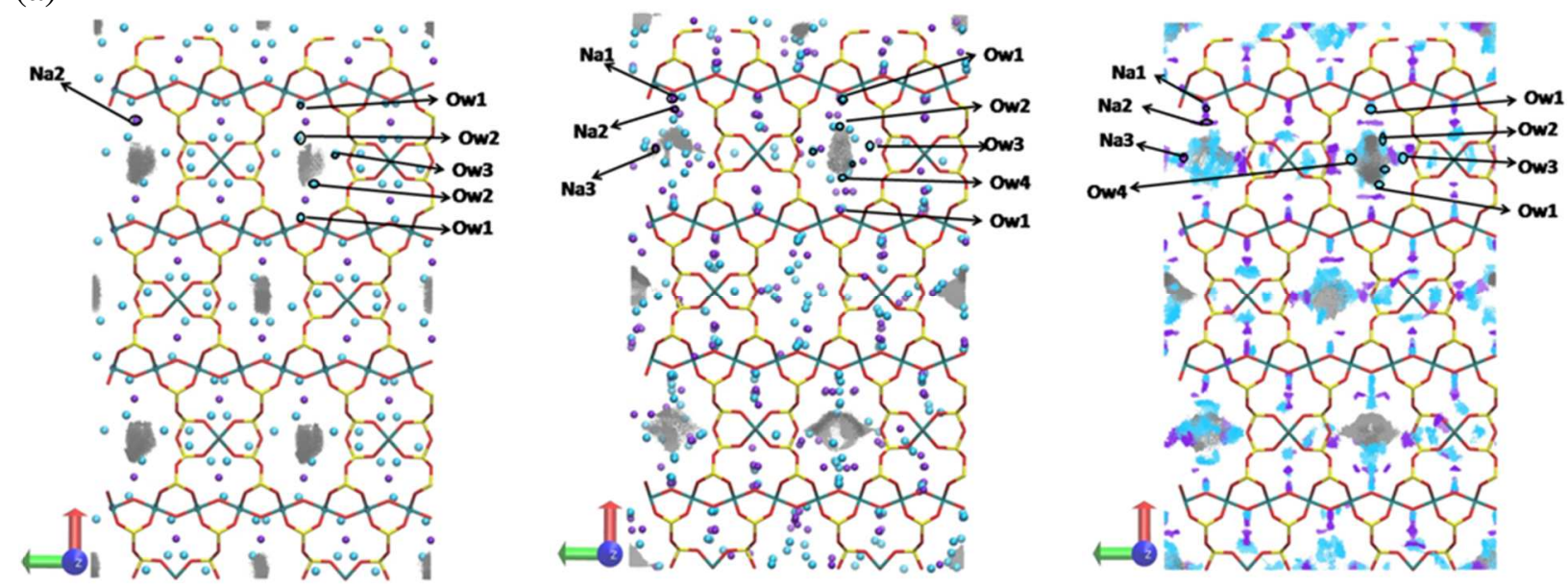

(b) 


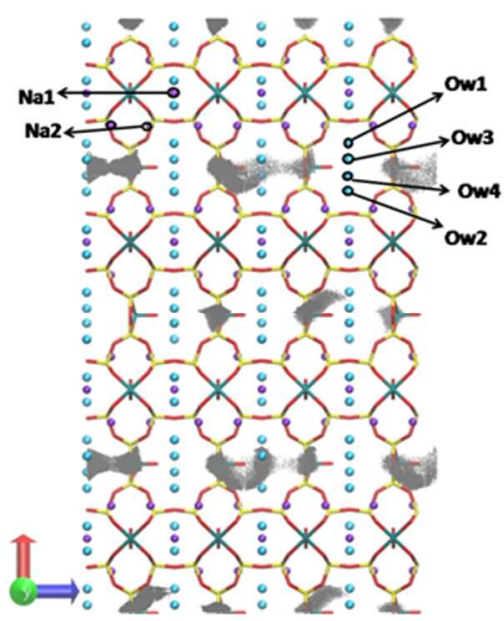

Strategy 1

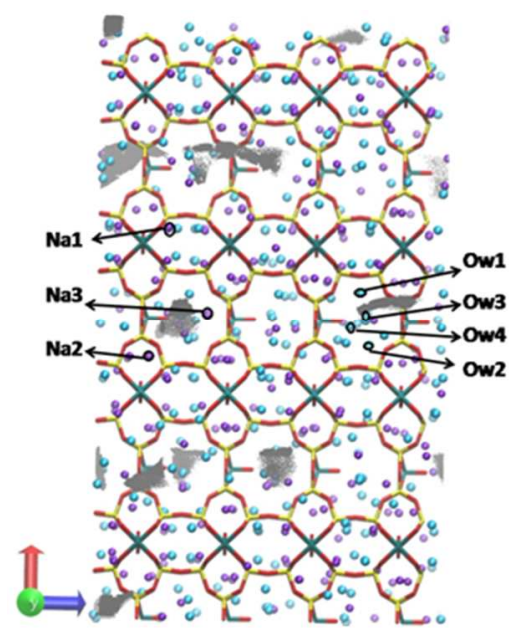

Strategy 2

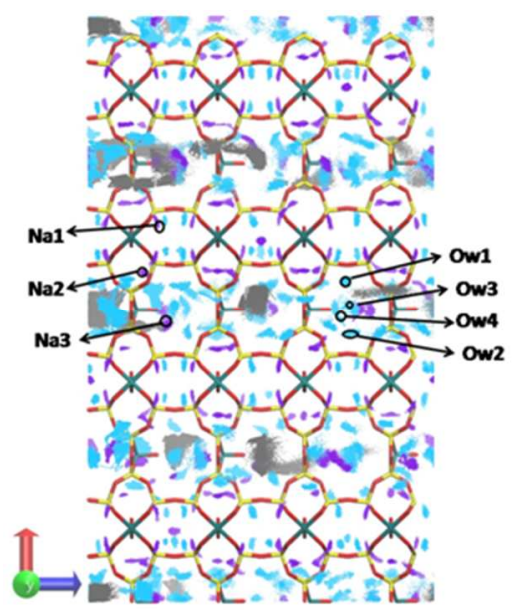

Strategy 3

Figure 11. Occupancy maps for $\mathrm{CH}_{4}$ at $300 \mathrm{~K}$ and $115 \mathrm{kPa}$ in the ABAB-AA-HB polymorph of ETS-4, shown in projections along the (a) $c$ and (b) $b$ vectors. For each direction, we compare results obtained using the three simulation strategies. Color coding is the same as in Figure 7.

Figure 12 compares the GCMC adsorption isotherms obtained in all 8 polymorphs considered in this work, for the three different simulation strategies. The first general impression is that the isotherms show a high degree of variability, so that the adsorbed amount at all pressures significantly depends on the precise polymorph structure. This variability is less pronounced for strategy 1, probably because the locations of cations and water from the crystal structure data already represent an average over several polymorph structures existing in real ETS-4 samples ${ }^{11}$. Conversely, strategy 2 leads to the highest variability because the extraframework species were "allowed" to find their most favorable positions during the preliminary NVT simulations. These favorable locations are generally distinct for each structure, which contributes to make the polymorphs even more different from each other. Strategy 3 shows intermediate behavior because, although starting from the same configurations as strategy 2 , cations and water molecules are allowed to adapt "on-the-fly" to the presence of adsorbed methane molecules. 
It is interesting to notice that the variability due to polymorph structure is even more significant than the effects of different treatments of cation and water mobility. In previous work, Siperstein et al. ${ }^{25}$ argued that the $\mathrm{ABCD}$ and $\mathrm{ABAB}$ stackings were not likely to significantly affect adsorption on ETS-4. Our results reveal that although this is true in some cases (compare isotherms for, e.g., $\mathrm{ABAB}-\mathrm{AA}-\mathrm{BV}$ and $\mathrm{ABCD}-\mathrm{AA}-\mathrm{BV}$ in Figure 12), differences can be significant for some polymorphs (compare isotherms for ABAB-AC-BV and ABCD-AC-BV in Figure 12). Differences between polymorphs with AA and AC stacking are also significant in some cases, but the most pronounced and consistent differences are between polymorphs of type BV and HB. Again, this can be understood by considering the different availability of the 8-MR pores in these two types of structure. In general, our results show that the polymorph structure needs to be taken into account for accurate adsorption predictions in ETS-4.

(a)

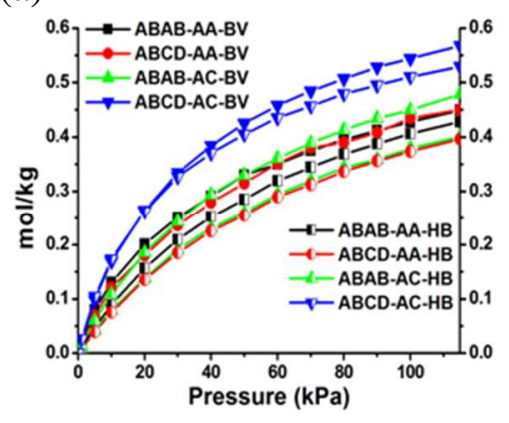

(b)

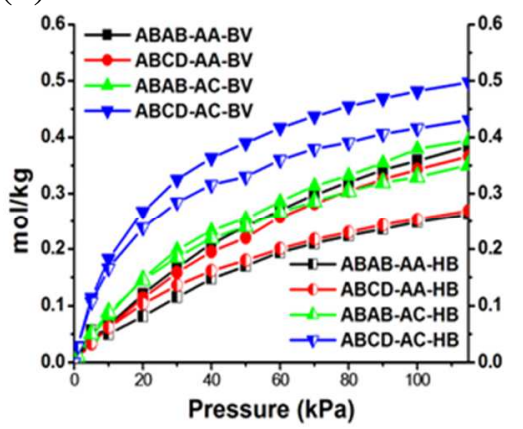

(c)

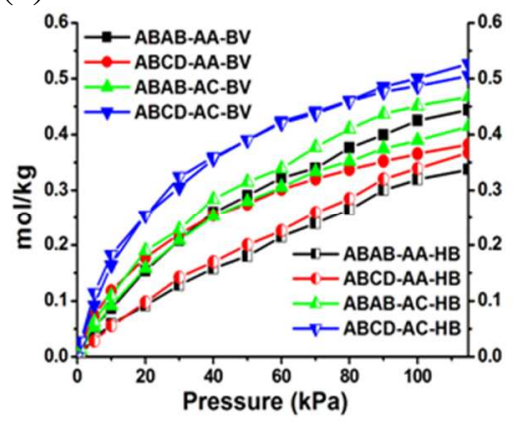

Figure 12. GCMC adsorption isotherms for $\mathrm{CH}_{4}$ in all different $\mathrm{NaETS}-4$ polymorphs at $303 \mathrm{~K}$ obtained by strategy 1 (a), strategy 2 (b) and strategy 3 (c).

So far, we have limited ourselves to calculating adsorption isotherms in pure ETS-4 polymorphs. However, real samples are almost certainly composed of different crystalline domains, each of them corresponding to a given polymorph. This degree of disorder is almost impossible to capture in a single simulation cell, but is likely to have profound effects on adsorption predictions, as suggested above for ETS-10. To try to account for the effects of this type of structural heterogeneity, we have adopted a rather simplified procedure to estimate an 
adsorption isotherm in a "real" sample of ETS-4. We simply assume that, on average, the adsorbed amount in the real sample can be approximated by the sum of adsorbed amounts in each crystalline sub-domain. This amounts to: i) assuming that each sub-domain is indeed adequately represented by a given pure polymorph; and ii) neglecting the effects of interfaces between sub-domains. Furthermore, in the absence of more detailed experimental information, we assume that each of the 8 pure polymorphs is equally likely to occur in the real sample.

Using these assumptions, we can estimate the adsorption isotherm for the real sample by simply taking the unweighted average of the 8 isotherms for the individual pure polymorphs. This approach is supported by the work of Braunbarth et al. ${ }^{8}$, who suggested that a "superposition structure" of all polymorphs of synthetic ETS-4 is likely the most valid one. The adsorption isotherms thus calculated are compared to experimental data ${ }^{24}$ at three different temperatures in Figure 13. It can be seen that all three different simulation strategies yield rather good agreement with experimental data, although the best results are obtained for strategy 3 (i.e., fully mobile cations and water). Apart from some systematic differences at the lowest temperature, where the simulated isotherms appear to be too favorable, the simulations correctly capture the curvature and the limiting capacity of the adsorption isotherms. Considering the oversimplified nature of the assumptions described above, and the fact that we are not employing any adjustable parameters, we find this agreement to be quite encouraging. Any discrepancies can probably be explained by a non-equitable distribution of polymorphs in the real sample - for example, if the sample contains a lower proportion of polymorphs $\mathrm{ABCD}-\mathrm{AC}-\mathrm{XX}$, the curvature of the isotherm at low pressure will be less pronounced, possibly leading to better agreement with experimental data. We did not further pursue these possibilities here. 
(a)

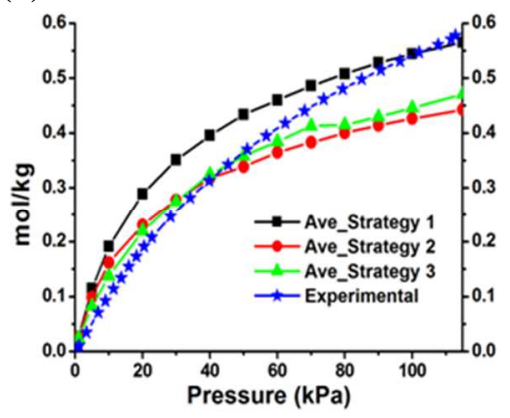

(b)

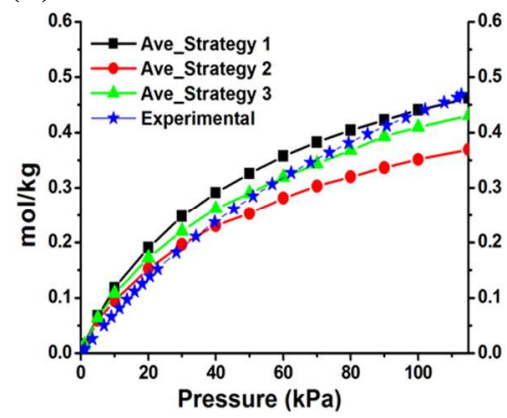

(c)

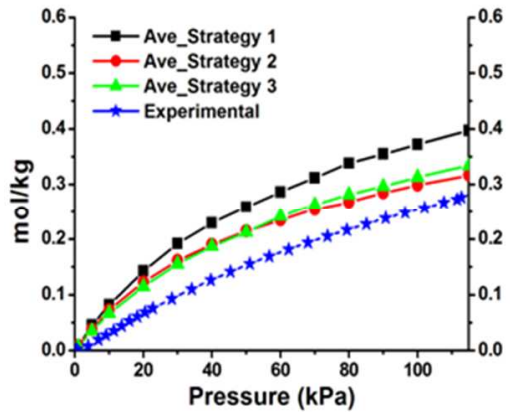

Figure 13. Comparison between experimental methane adsorption isotherms in Na-ETS-4 at 288 $\mathrm{K}$ (left), $303 \mathrm{~K}$ (middle) and $318 \mathrm{~K}$ (right), and simulated isotherms obtained by averaging over individual GCMC isotherms for each different polymorph structure.

As a final comparison, we report the heat of adsorption of methane in ETS-4 in Table 1, for each individual polymorph and each simulation strategy. Isosteric heats of adsorption were calculated from adsorption isotherms collected at different temperatures, using the ClausiusClapeyron equation,

$$
-\Delta_{a d} H^{o}=R[\partial \ln P / \partial(1 / T)]_{\theta}
$$

Where $R$ is the universal gas constant, $\theta$ is the fractional coverage at pressure $P$ and temperature $T$. If we again assume that the real material is a uniform average of the eight polymorphs, the average heats of adsorption are $-31.4 \mathrm{~kJ} / \mathrm{mol},-28.7 \mathrm{~kJ} / \mathrm{mol}$ and $-28.0 \mathrm{~kJ} / \mathrm{mol}$ for strategies 1,2 and 3, respectively, with extreme heats of adsorption differing more from the average value in the case of strategy $1(-5.1$ and $+4.7 \mathrm{~kJ} / \mathrm{mol})$ than in strategies $2(-3.5$ and $+1.9 \mathrm{~kJ} / \mathrm{mol})$ and $3(-$ 1.5 and $+1.9 \mathrm{~kJ} / \mathrm{mol})$. The average heats of adsorption compare very well with the experimental value of $-29.3 \mathrm{~kJ} / \mathrm{mol}^{24}$, particularly for strategies 2 and 3 . This provides further support for our conclusion that the effects of both structural heterogeneity and mobility of extra-framework 
species should be taken into account in adsorption predictions by molecular simulation for ETS materials.

Table 1. Heat of adsorption of methane in different Na-ETS-4 polymorphs.

\begin{tabular}{|c|c|c|c|}
\hline Na-ETS-4 polymorph & Strategy 1 & Strategy 2 & Strategy 3 \\
\hline ABAB-AA-BV & -33.4 & -31.2 & -28.6 \\
\hline ABCD-AA-BV & -28.9 & -28.8 & -28.6 \\
\hline ABAB-AC-BV & -28.7 & -27.1 & -27.2 \\
\hline ABCD-AC-BV & -36.5 & -30.1 & -29.3 \\
\hline ABAB-AA-HB & -30.1 & -29.2 & -26.1 \\
\hline ABCD-AA-HB & -26.7 & -26.8 & -27.3 \\
\hline ABAB-AC-HB & -31.6 & -26.8 & -29.5 \\
\hline ABCD-AC-HB & -35.3 & -29.5 & \\
\hline
\end{tabular}

${ }^{\mathrm{a}}$ Experimental heat of adsorption for $\mathrm{CH}_{4}$ in Na-ETS-4 is $-29.3 \mathrm{~kJ} / \mathrm{mol}$ (ref. ${ }^{24}$ )

\section{Conclusions}

Grand canonical Monte Carlo simulations with extra-framework cations and water molecules were carried out to elucidate the influence of their locations and mobility in the simulation of methane adsorption in the channels of Engelhard titanosilicates, ETS-10 and ETS-

4. By allowing the cations and water to equilibrate during NVT simulations of the empty structure (i.e., without adsorbed methane), we have observed that some of these extra-framework species migrate to different regions of the pore space than predicted in single crystal data. This is at least partly due to the high degree of uncertainty in the experimental diffraction data when it comes to mobile species, as well as to the fact that diffraction data are providing locations that are averaged over different structural polymorphs of the titanosilicate materials. Indeed, in most 
cases studied, simulations of methane adsorption with cations/water fixed at their single crystal positions gave significantly different results than when these species were allowed to move. An intermediate approach, namely fixing the cations/water at the most favorable positions determined from preliminary NVT simulations, normally yielded very similar results to the isotherms considering full mobility. However, in some of the ETS-4 polymorphs, cations and water were seen to migrate "on-the-fly" in response to methane adsorption, a behavior that was not captured in any of the simulations with fixed extra-framework species. This suggests that even for simple non-polar gases like methane, cation and water mobility during adsorption should be taken into account in simulations to provide a realistic description of the adsorption process.

We compared our simulated adsorption isotherms to experimental data for methane adsorption in ETS-10 at 5 different temperatures. Although the simulations always overestimate adsorption, this could be due to the presence of impurities, defects and/or excluded pore volume in the real materials. Indeed, when experimental data are rescaled by an empirical factor, agreement with simulations is reasonable, suggesting that this can at least partly explain the observed discrepancies. Nevertheless, at the moment we cannot exclude inaccuracies in the molecular model as a possible source of error, and additional studies are needed to clarify this issue. In particular, interaction energies between adsorbates and the extra-framework cations should be the subject of a more detailed parameterization study, as this is currently an unsolved problem even for zeolite materials.

For ETS-4, we simulated adsorption in eight different pure polymorph structures, obtained by combining different unit cell stackings and different arrangements of apical TiO units. Simulated isotherms show a very significant dependence on the structural arrangement of 
the framework, with the largest differences being observed when the distribution of apical oxygen atoms was changed. Indeed, structures in which all eight-member-ring pores were partially blocked by oxygen atoms showed the most significant effects of cation and water mobility. This can be understood by noticing that even a very small amount of water or cations present in these pores will completely exclude methane from these regions of the pore space. As real samples of ETS-4 are likely to contain a variety of crystalline domains with different polymorph structures, we applied a simple averaging procedure to estimate the composite adsorption isotherm in a possible real sample of ETS-4. These isotherms compared reasonably well with experimental data at three different temperatures, as did the predicted isosteric heat of adsorption, considering the high degree of heterogeneity of these materials. This was particularly so for the simulations with fully mobile water and cations, further supporting our claim that mobility of these species plays an important role in adsorption.

Although they are fundamentally crystalline materials, titanosilicates pose particular challenges to modelling studies because of their high degree of heterogeneity. This arises not only from heterogeneities in the framework structure itself (e.g., defects, polymorphism, etc.), but also from the presence of relatively mobile extra-framework species, such as cations and water. Our simulations in these systems show that all these sources of heterogeneity must be taken into account in order to yield reliable adsorption predictions. Despite the fact that our simulations provide at least good qualitative agreement with experiments, additional studies are needed to develop a more robust strategy for describing structural heterogeneity in ETS materials. 


\section{Supporting Information Available}

Detailed description of computational methodology used for methane GCMC simulation in ETS materails (Figures S1-S2 and Table S1). Procedures adopted to find equilibration as well as optimal extra framework species positions in ETS materials (Figures S3-S5). Comparison of sodium and water positions in all ETS-4 polymorphs obtained from experimental crystallographic positions, instantaneous equilibrated sodium cation and water locations obtained from NVT simulations (Figure S6). GCMC adsorption isotherms (Figures S7-S9) and full occupancy plots (Figures S10-S12) for $\mathrm{CH}_{4}$ in all different Na-ETS-4 polymorphs at different temperatures obtained by strategies 1, 2 and 3. Comparison of adsorption isotherms on ETS-4 obtained with two different water models (Figure S13). This information is available free of charge via the Internet at http://pubs.acs.org/.

\section{Acknowledgments}

This work is supported by projects PTDC/EQU-EQU/099423/2008, FCOMP-01-0124-FEDER037271, PEst-C/CTM/LA0011/2013 and Programa Investigador FCT, financed by FEDER through Programa Operacional Factores de Competitividade, COMPETE, and by FCT (Fundação para a Ciência e a Tecnologia) funds. RSP gratefully acknowledges a post-doctoral fellowship from FCT with reference SFRH/BPD/70283/2010.

\section{References}

1. Ruthven, D. M. Principles of Adsorption and Adsorption Processes Wiley New York, 1984.

2. Jasra, R. V.; Choudary, N. V.; Bhat, S. G. T. Separation of Gases by Pressure Swing Adsorption. Separ. Sci. and Technol. 1991, 246, 885-930. 
3. Grande, C. A.; Lopes, F. V. S.; Ribeiro, A. M.; Loureiro, J. M.; Rodrigues, A. E. Adsorption of off-gases from steam methane reforming $\left(\mathrm{H}_{2}, \mathrm{CO}_{2}, \mathrm{CH}_{4}, \mathrm{CO}\right.$ and $\left.\mathrm{N}_{2}\right)$ on activated carbon. Separ. Sci. and Technol. 2008, 43, 1338-1364.

4. Dabrowski, N.; Windmeier, C.; Oellrich, L. R. Purification of Natural Gases with High $\mathrm{CO}_{2}$ Content Using Gas Hydrates. Energ. Fuel 2009, 23, 5603-5610.

5. Peng, X. A.; Cao, D. P.; Wang, W. C. Computational Study on Purification of $\mathrm{CO}_{2}$ from Natural Gas by C-60 Intercalated Graphite. Ind. Eng. Chem. Res. 2010, 49, 8787-8796.

6. Baowan, D. Modelling of carbon dioxide: methane separation using titanium dioxide nanotubes. J. Math. Chem. 2012, 50, 300-309.

7. Kuznicki, S. M. Preparation of small-pored crystalline titanium molecular sieve zeolites, US patent, US4938939 1990.

8. Braunbarth, C.; Hillhouse, H. W.; Nair, S.; Tsapatsis, M.; Burton, A.; Lobo, R. F.; Jacubinas, R. M.; Kuznicki, S. M. Structure of strontium ion-exchanged ETS-4 microporous molecular sieves. Chem. Mater. 2000, 12, 1857-1865.

9. Majumdar, B.; Bhadra, S. J.; Marathe, R. P.; Farooq, S. Adsorption and Diffusion of Methane and Nitrogen in Barium Exchanged ETS-4. Ind. Eng. Chem. Res. 2011, 50, 30213034.

10. Kuznicki, S. M.; Bell, V. A.; Nair, S.; Hillhouse, H. W.; Jacubinas, R. M.; Braunbarth, C. M.; Toby, B. H.; Tsapatsis, M. A titanosilicate molecular sieve with adjustable pores for sizeselective adsorption of molecules. Nature 2001, 412, 720-724.

11. Nair, S.; Jeong, H. K.; Chandrasekaran, A.; Braunbarth, C. M.; Tsapatsis, M.; Kuznicki, S. M. Synthesis and structure determination of ETS-4 single crystals. Chem. Mater. 2001, 13, 4247-4254.

12. Nair, S.; Tsapatsis, M.; Toby, B. H.; Kuznicki, S. M. A study of heat-treatment induced framework contraction in strontium-ETS-4 by powder neutron diffraction and vibrational spectroscopy. J. Am. Chem. Soc. 2001, 123, 12781-12790.

13. Kuznicki, S. M.; Petrovich, I.; Desai, B. T. Small pored crystalline titanium molecular sieve zeolites and their use in gas separation process US patent, US6068682 2000.

14. Marathe, R. P.; Mantri, K.; Srinivasan, M. P.; Farooq, S. Effect of ion exchange and dehydration temperature on the adsorption and diffusion of gases in ETS-4. Ind. Eng. Chem. Res. 2004, 43, 5281-5290.

15. Marathe, R. P. Investigating entropy changes during gas adsorption in ETS-4. J. Colloid Interf. Sci. 2005, 290, 69-75.

16. Marathe, R. P.; Farooq, S.; Srinivasan, M. P. Modeling gas adsorption and transport in smallpore titanium silicates. Langmuir 2005, 21, 4532-4546.

17. Marathe, R. P.; Farooq, S.; Srinivasan, M. P. Effects of site occupancy, cation relocation, and pore geometry on adsorption kinetics in ETS-4. J. Phys. Chem. B 2005, 109, 3257-3261.

18. Philippou, A.; Rocha, J.; Anderson, M. W. The strong basicity of the microporous titanosilicate ETS-10. Catal. Lett. 1999, 57, 151-153.

19. Bordiga, S.; Palomino, G. T.; Zecchina, A.; Ranghino, G.; Giamello, E.; Lamberti, C. Stoichiometric and sodium-doped titanium silicate molecular sieve containing atomically defined -OTiOTiO- chains: Quantum ab initio calculations, spectroscopic properties, and reactivity. J. Chem. Phys. 2000, 112, 3859-3867.

20. Krishna, R. M.; Prakash, A. M.; Kurshev, V.; Kevan, L. Photoinduced charge separation of methylphenothiazine in microporous titanosilicate M-ETS-10 $\left(\mathrm{M}=\mathrm{Na}^{+}+\mathrm{K}^{+}, \mathrm{H}^{+}, \mathrm{Li}^{+}, \mathrm{Na}^{+}\right.$, 
$\left.\mathrm{K}^{+}, \mathrm{Ni}^{2+}, \mathrm{Cu}^{2+}, \mathrm{Co}^{2+}\right)$ and $\mathrm{Na}^{+}, \mathrm{K}^{+}$-ETS-4 molecular sieves at room temperature. Phys. Chem. Chem. Phys. 1999, 1, 4119-4124.

21. Pinto, M. L.; Rocha, J.; Gomes, J. R. B.; Pires, J. Slow Release of NO by Microporous Titanosilicate ETS-4. J. Am. Chem. Soc. 2011, 133, 6396-6402.

22. Kuznicki, S. M. Large-pored crystalline titanium molecular sieve zeolites. US patent, US4453202 1989.

23. Marathe, R. P.; Srinivasan, M. P.; Farooq, S. Effect of Si : Ti ratio on energetic heterogeneity in ETS-4. Chem. Eng. Sci. 2004, 59, 6021-6025.

24. Pillai, R. S.; Peter, S. A.; Jasra, R. V. Adsorption of carbon dioxide, methane, nitrogen, oxygen and argon in NaETS-4. Micropor. Mesopor. Mat. 2008, 113, 268-276.

25. Siperstein, F. R.; Lisal, M.; Brennan, J. K. A Molecular Simulation Study of Adsorption of Nitrogen and Methane in Titanium Silicate (ETS-4). Collect. Czech. Chem. C 2010, 75, 145164.

26. Bhadra, S. J.; Farooq, S. Separation of Methane-Nitrogen Mixture by Pressure Swing Adsorption for Natural Gas Upgrading. Ind. Eng. Chem. Res. 2011, 50, 14030-14045.

27. Smit, B.; Maesen, T. L. M. Molecular simulations of zeolites: Adsorption, diffusion, and shape selectivity. Chem. Rev. 2008, 108, 4125-4184.

28. Mitchell, M. C.; Gallo, M.; Nenoff, T. M. Computer simulations of adsorption and diffusion for binary mixtures of methane and hydrogen in titanosilicates. J. Chem. Phys. 2004, 121, 1910-1916.

29. Gallo, M.; Nenoff, T. M.; Mitchell, M. C. Selectivities for binary mixtures of hydrogen/methane and hydrogen/carbon dioxide in silicalite and ETS-10 by Grand Canonical Monte Carlo techniques. Fluid Phase Equilibr. 2006, 247, 135-142.

30. Nalaparaju, A.; Zhao, X. S.; Jiang, J. W. Molecular interplay of cations and nonpolar/polar sorbates in titanosilicate ETS-10. J. Phys. Chem. C 2008, 112, 12861-12868.

31. Wang, X. Q.; Jacobson, A. J. Crystal structure of the microporous titanosilicate ETS-10 refined from single crystal X-ray diffraction data. Chem. Commun. 1999, 973-974.

32. Gupta, A.; Chempath, S.; Sanborn, M. J.; Clark, L. A.; Snurr, R. Q. Object-oriented programming paradigms for molecular modeling. Mol. Simul. 2003, 29, 29-46.

33. Chempath, S.; Snurr, R. Q.; Low, J. J. Molecular modeling of binary liquid-phase adsorption of aromatics in silicalite. AIChE J. 2004, 50, 463-469.

34. Rappe, A. K.; Casewit, C. J.; Colwell, K. S.; Goddard, W. A.; Skiff, W. M. UFF, a Full Periodic-Table Force-Field for Molecular Mechanics and Molecular-Dynamics Simulations. J Am. Chem. Soc. 1992, 114, 10024-10035.

35. Martin, M. G.; Siepmann, J. I. Transferable potentials for phase equilibria. 1. United-atom description of n-alkanes. J. Phys. Chem. B 1998, 102, 2569-2577.

36. Jorgensen, W. L.; Chandrasekhar, J.; Madura, J. D.; Impey, R. W.; Klein, M. L. Comparison of Simple Potential Functions for Simulating Liquid Water. J. Chem. Phys. 1983, 79, 926935.

37. Al-Baghli, N. A.; Loughlin, K. F. Adsorption of methane, ethane, and ethylene on titanosilicate ETS-10 zeolite. J. Chem. Eng. Data. 2005, 50, 843-848.

38. Anderson, M. W.; Terasaki, O.; Ohsuna, T.; Philippou, A.; Mackay, S. P.; Ferreira, A.; Rocha, J.; Lidin, S. Structure of the Microporous Titanosilicate ETS-10. Nature 1994, 367, 347-351. 
39. Anderson, M. W.; Terasaki, O.; Ohsuna, T.; Malley, P. J. O.; Philippou, A.; Mackay, S. P.; Ferreira, A.; Rocha, J.; Lidin, S. Microporous Titanosilicate Ets-10 - a Structural Survey. Philos. Mag. B 1995, 71, 813-841.

40. Das, T. K.; Chandwadkar, A. J.; Sivasanker, S. A rapid method of synthesizing the titanium silicate ETS-10. Chem. Commun. 1996, 1105-1106.

41. Sankar, G.; Bell, R. G.; Thomas, J. M.; Anderson, M. W.; Wright, P. A.; Rocha, J. Determination of the Structure of Distorted $\mathrm{TiO}_{6}$ Units in the Titanosilicate ETS-10 by a Combination of X-ray Absorption Spectroscopy and Computer Modeling. J. Phys. Chem. 1996, 100, 449-452.

42. Pavel, C. C.; Park, S.-H.; Dreier, A.; Tesche, B.; Schmidt, W. Structural Defects Induced in ETS-10 by Postsynthesis Treatment with $\mathrm{H}_{2} \mathrm{O}_{2}$ Solution. Chem. Mater. 2006, 18, 3813-3820.

43. Jorge, M.; Fischer, M.; Gomes, J. R. B.; Siquet, C.; Santos, J. C.; Rodrigues, A. E. Accurate Model for Predicting Adsorption of Olefins and Paraffins on MOFs with Open Metal Sites. Ind. Eng. Chem. Res. 2014, DOI:10.1021/ie500310c. 
TOC Graphic

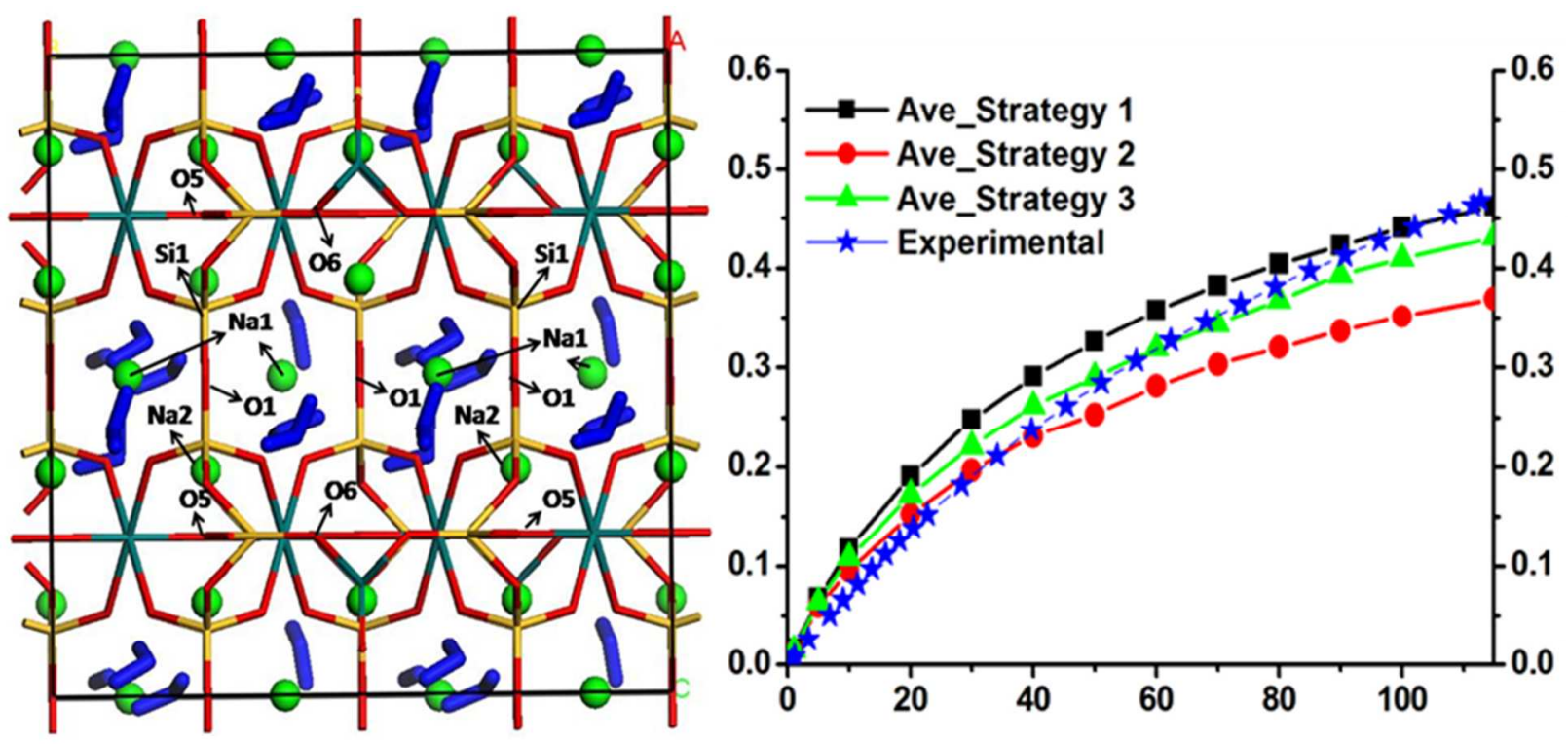

